\title{
The Mediating Effect of Sleep Quality on the Relationship between PTSD and Physical Activity
}

\author{
Lisa S. Talbot, Ph.D. ${ }^{1,2}$; Thomas C. Neylan, M.D., F.A.A.S.M. ${ }^{1,2}$; Thomas J. Metzler, M.S. ${ }^{1 ;}$ Beth E. Cohen, M.D., M.A.S. ${ }^{1,3}$ \\ ${ }^{I}$ San Francisco VA Medical Center, San Francisco, CA; ${ }^{2}$ Department of Psychiatry, University of California, San Francisco, CA; \\ ${ }^{3}$ Department of Medicine, University of California, San Francisco, CA
}

Study Objectives: Physical inactivity is linked to health outcomes such as obesity, diabetes, and psychiatric disorders. Sleep disturbance has been linked to the same adverse outcomes. We examine the influence of sleep on physical activity as a novel approach to understand these relationships. Specifically, our objective was to determine whether low sleep quality predicts low physical activity in posttraumatic stress disorder (PTSD), a disorder associated with sleep disturbance, physical inactivity, and poor health outcomes.

Methods: We used data from the Mind Your Heart Study, a prospective cohort study of 736 outpatients recruited from two Department of Veterans Affairs (VA) medical centers. We assessed PTSD with the Clinician Administered PTSD Scale, sleep quality using an item from the Pittsburgh Sleep Quality Index, and physical activity by self-report at baseline and again one year later. Hierarchical multiple regression models and structural equation modeling were used to examine the relationships among PTSD, sleep, and physical activity. Results: Sleep quality but not PTSD status was prospectively associated with lower physical activity in a model adjusting for age, sex, apnea probability, depression, body mass index, and baseline physical activity $(\beta=0.129$, SE $=0.072, p<0.01)$. Structural equation modeling indicated that the results were consistent with sleep quality statistically mediating the relationship between PTSD status at baseline and physical activity one year later.

Conclusions: Worse sleep quality predicts lower physical activity in PTSD, providing possible evidence for a behavioral pathway from disturbed sleep to poor physical health outcomes. Keywords: posttraumatic stress disorder, sleep quality, physical activity

Citation: Talbot LS, Neylan TC, Metzler TJ, Cohen BE. The mediating effect of sleep quality on the relationship between PTSD and physical activity. J Clin Sleep Med 2014;10(7):795801.
Dhysical activity is associated with a range of outcomes 1 relevant to psychiatric disorders. For example, regular physical activity is associated with lower rates of numerous disorders including depression, panic disorder, agoraphobia, social phobia, and specific phobia'; a large body of evidence indicates that exercise improves anxiety and depression ${ }^{2}$; and accruing evidence suggests that aerobic exercise is associated with neurogenesis and improved memory function. ${ }^{3}$ A recent study also demonstrated that the association between depressive symptoms and cardiovascular events in a sample of 1,000 outpatients with coronary artery disease was largely explained by self-reported physical inactivity. ${ }^{4}$

Studies suggest that sleep may be a predictor of physical activity. In general, more sleep is associated with increased levels of physical activity, ${ }^{5,6}$ although one epidemiological study did not see this association. ${ }^{7}$ While more research has assessed the influence of physical activity on sleep, the relationship between the two factors has been proposed to be bidirectional, ${ }^{8}$ and a growing literature supports the effects of sleep on physical activity. For example, a recent study of 11 women with insomnia tracked for 16 weeks demonstrated that sleep influenced next day exercise (i.e., longer sleep onset was associated with shorter next-day exercise duration); interestingly, in this sample, exercise did not have an effect on subsequent sleep. ${ }^{8}$ An observational study of adolescents

\section{BRIEF SUMMARY \\ Current Knowledge/Study Rationale: Both physical activity and sleep have been linked to health outcomes such as obesity. We examined the influence of sleep on physical activity as a novel approach to under- stand these relationships, particularly in posttraumatic stress disorder, a disorder associated with sleep disturbance, physical inactivity, and poor health outcomes. \\ Study Impact: Worse sleep quality predicts lower physical activity in PTSD, providing possible evidence for a behavioral pathway from dis- turbed sleep to poor physical health outcomes.}

indicated that poor sleep quality (rather than sleep duration) was associated with decreased physical activity measured using accelerometry. ${ }^{9}$ A few small laboratory studies have also examined the influence of sleep on physical activity. Some of these studies have yielded discordant findings, ${ }^{10}$ perhaps because the experimental studies on this topic have generally relied on acute sleep deprivation. It is possible that the forced short sleep duration characteristic of these studies may not represent the chronic sleep disturbance that is experienced by many individuals in daily life (e.g., longer sleep duration but with fragmentation resulting in reduced sleep quality). In addition, these studies typically only capture next-day activity, whereas many individuals may engage in physical activity several times per week but not daily. A recent longer study 
involving a small sample of healthy, non obese adults with a family history of type 2 diabetes found that one week of sleep restriction resulted in less physical activity and more sedentary time compared to a matched period with sufficient sleep opportunity. ${ }^{11}$

Notably, sleep has numerous dimensions such as duration, continuity, timing within the 24-hour day, alertness/sleepiness, and satisfaction/quality. ${ }^{12}$ The sleep-physical activity literature has focused on several different dimensions. For example, the previously cited studies focused on sleep duration, poor sleep quality (i.e., a subjective assessment of sleep), or sleep disturbance broadly, which can include sleep discontinuity. In the present study, we focus on sleep quality given (a) the importance of subjective satisfaction or dissatisfaction with sleep (i.e., it leads individuals to seek treatment); (b) its relationship to objective measures (e.g., slow wave sleep ${ }^{13}$ ); and (c) its connection to poor health outcomes. ${ }^{14,15}$

Indeed, sleep disturbance across all of its dimensions has been linked to the same adverse physical health outcomes as physical inactivity. For example, sleep duration has been prospectively associated with weight gain, while poor sleep quality has been associated with metabolic syndrome, hypertension, and coronary heart disease. ${ }^{14-19}$ Neuroendocrine changes caused by poor sleep partially account for these relationships, ${ }^{20}$ but behavioral factors related to sleep disturbance, such as decreased physical activity, may also play a role.

Given that sleep disturbance and physical inactivity are associated with the same poor health outcomes and recent evidence suggests poor sleep predicts physical inactivity, we use a prospective design to evaluate whether sleep quality predicts physical activity in individuals with PTSD. We specifically examine this question in PTSD because the disorder is strongly linked to sleep disturbance, physical inactivity, and poor physical health outcomes. In terms of sleep, poor sleep quality is characteristic of PTSD, with sleep disturbance being the most frequently reported symptom of PTSD. ${ }^{21-23}$ Moreover, self-reported poor sleep quality in PTSD appears to be minimally influenced by age, gender, and psychiatric comorbidity. ${ }^{24}$ Several studies also suggests that individuals with PTSD demonstrate lower rates of exercise compared to others without PTSD and that the majority of individuals with PTSD exercise infrequently. ${ }^{25-27}$ Additionally, rates of physical activity appear to decrease following the onset of PTSD. ${ }^{28}$ Finally, posttraumatic stress disorder (PTSD) is associated with poor physical health outcomes, including obesity, metabolic syndrome, and cardiovascular disease. ${ }^{29-31}$

There is a lack of prospective research examining sleep and physical activity in PTSD, and disentangling these relationships could serve as a first step to developing targeted behavioral interventions to prevent morbidity and mortality in PTSD. In the current study, we assessed the relationship between sleep quality at baseline and physical activity one year later in individuals with and without PTSD. We hypothesized that PTSD would be associated with lower sleep quality and that both PTSD and sleep quality at baseline would predict lower physical activity one year later. We further hypothesized that the association between PTSD and physical activity one year later would be explained partially or fully by sleep quality.

\section{METHODS}

\section{Participants}

The Mind Your Heart Study is a prospective cohort study designed to examine the association between PTSD and health outcomes. Participants were recruited between February 2008 and June 2010 from two Department of Veterans Affairs (VA) Medical Centers (San Francisco VA Medical Center and the VA Palo Alto Health Care System, California). Potential participants were excluded if they planned on leaving the area in 3 years or did not have contact information for follow-up. Potential participants were also excluded if they were unable to walk one block or had a myocardial infarction in the prior 6 months, as a cardiac treadmill test was done for the larger study and could be contraindicated in these cases. All participants provided written informed consent and appropriate institutional review boards approved the research protocol.

Overall, 1,020 VA patients were assessed for eligibility. One hundred four $(10.2 \%)$ were found ineligible, primarily due to lack of contact information for follow-up $(\mathrm{n}=82)$. Of the remaining 916 eligible patients, $170(18.6 \%)$ declined to participate or did not complete the baseline consent process, such that 746 participants were ultimately enrolled in the study. Ten participants were excluded from these analyses because they did not complete full PTSD assessments or because the supervising study psychologist had concerns about the accuracy of the PTSD diagnosis. Fifty participants did not complete the one-year follow-up by phone; of this group, 10 had died.

\section{PTSD}

We evaluated PTSD with the Clinician-Administered PTSD Scale (CAPS) ${ }^{32}$ using criteria from the Diagnostic and Statistical Manual of Mental Disorders IV (DSM-IV). The CAPS is the most widely used structured interview for diagnosing PTSD and has excellent test-retest reliability $(r=0.92-0.99)$ and internal consistency $(\alpha=0.80-0.90){ }^{32,33}$ The PTSD group consisted of subjects with either full or subsyndromal PTSD, as subsyndromal PTSD is associated with significant impairment in health and functioning. ${ }^{34}$ Subsyndromal PTSD was defined as meeting diagnostic criteria for the reexperiencing cluster and either avoidance or hyperarousal clusters, in addition to the other CAPS criteria. ${ }^{35}$ We also required this group to exhibit symptoms meeting a total CAPS score $>40$, as defined by the authors of the CAPS as signifying moderate or threshold PTSD. ${ }^{33}$ Two hundred fifty-eight individuals met criteria for full or subsyndromal PTSD.

\section{Sleep}

We assessed sleep by asking participants to rate their sleep quality overall during the last month using a single rating from 0 ("very good") to 4 ("very bad"). Sleep quality was coded as a continuous variable, and items were reversed scored prior to analyses, such that a higher score reflected better sleep quality. The question was derived and modified from the Pittsburgh Sleep Quality Index (PSQI), ${ }^{36}$ a self-rated questionnaire which assesses sleep quality and disturbances. The published version of the PSQI uses a 0-3 scale, but in the present study an expanded 0-4 scale for the sleep quality item was adopted. The 
global PSQI has an overall reliability coefficient of 0.83 , and the test-retest reliability showed no significant difference was found between two separated occasions. ${ }^{36}$ Self-reported sleep quality is an important clinical construct which involves a subjective assessment of sleep that has more specifically been defined by individuals with and without sleep problems as relating to tiredness on waking and throughout the day, feeling rested and restored on waking, and the number of awakenings experienced in the night. ${ }^{37} \mathrm{~A}$ question assessing sleep quality has been recommended as essential when assessing sleep by self-report, ${ }^{38}$ and the particular sleep quality question used in the present study has previously predicted outcomes such as anger expression. ${ }^{39}$

\section{Physical Activity}

To assess baseline physical activity, we asked participants, "Which of the following statements best describes how physically active you have been during the last month, that is, doing activities such as 15 to 20 minutes of brisk walking, swimming, general conditioning, or recreational sports?" Participants chose from one of the following 6 categories: not at all active, a little active (1-2 times per month), fairly active (3-4 times per month), quite active (1-2 times per week), very active (3-4 times per week), or extremely active (5 or more times per week). Self-report assessment of physical activity is reliable and valid, and single-response items have demonstrated excellent construct validity. ${ }^{40,41}$ In particular, this item was the strongest mediator of the association of depression and recurrent cardiovascular events in a previous study. ${ }^{4}$ Participants again answered this question during the telephone follow-up interview one year later.

\section{Covariates}

We administered a self-report demographics questionnaire to all participants to determine age, sex, ethnicity, income, education, and medical history. ${ }^{4}$ We assessed sleep apnea probability using a validated multivariate apnea risk index. ${ }^{42}$ The index was primarily derived from 3 self-report items assessing sleep disordered breathing (snorting or gasping, loud snoring, and breathing stops/choking/struggling for breath). The 3 questions have test-retest correlations between a 2 -week period of 0.92 $(\mathrm{p}<0.0001)$ and high internal consistency (Cronbach $\alpha=0.85$ to 0.93$){ }^{42}$ The multivariate apnea index also included age, gender, and body mass index (BMI). ${ }^{42}$ We used the Alcohol Use Disorders Identification Test-Consumption Questions (AUDITC), a validated screening questionnaire, to assess possible problematic alcohol use. ${ }^{43}$ We measured height and weight using standardized protocols, and BMI was calculated by dividing weight in kilograms by the square of height in meters. The Patient Health Questionnaire (PHQ-9) ${ }^{44}$ was used to evaluate depressive symptoms. This self-report instrument measures the frequency of depressive symptoms corresponding to the 9 symptom criteria in the DSM-IV. A standard cut-point $\geq 10$ is used to define depression and has demonstrated excellent validity when compared with a mental health interview, with a sensitivity of $88 \%$ and a specificity of $88 \%{ }^{44}$

\section{Statistical Analyses}

Our objectives were to evaluate the association of PTSD with sleep quality and the interrelationships among PTSD, sleep quality, and physical activity one year later. We compared differences in characteristics between participants with and without PTSD using $t$-tests for continuous variables and $\chi^{2}$ tests for dichotomous variables. Hierarchical multiple regression models were used to examine the relationships among sleep quality, PTSD, and predictors of physical activity. Models assessing the relationship between PTSD and sleep quality were first adjusted for age and sex to ensure that any observed differences were not simply due to basic demographic differences. We then adjusted for baseline physical activity, apnea probability, depression status, and BMI, as these variables differed between participants with and without PTSD. Similarly, models assessing the predictors of year 1 physical activity were first adjusted for age and sex and then for baseline physical activity, apnea probability, depression status, and BMI. These analyses were performed with IBM SPSS 19, Release Version 19.0 (2010, Armonk, NY), and all statistical tests were two-sided with $\alpha=0.05$.

Mediation analyses were conducted to determine the potential mediating effect of sleep quality on the relationship between PTSD status and physical activity one year later. Specifically, path analyses via a structural equation modeling framework and bootstrap estimation were performed. Participants were excluded from the mediation analyses if they did not have complete data on physical activity at baseline, sleep quality at baseline, and physical activity at year 1 follow-up. Few participants were excluded, and exclusion did not differ by PTSD status $(n=24[9.3 \%]$ participants with PTSD were excluded compared to $\mathrm{n}=46[9.6 \%]$ participants without PTSD). The analysis was performed with SPSS Amos, Release Version 20.0 (2010, Armonk, NY), and statistical tests were two-sided with $\alpha=0.05$.

\section{RESULTS}

\section{Participants}

Of the 736 military veteran participants, 258 (35\%) had current or subsyndromal PTSD. Compared with participants who did not have PTSD, those with PTSD were significantly more likely to be female, have current depression, have a higher probability of sleep apnea, have a higher BMI, and have lower levels of baseline physical activity (Table 1). We did not find significant differences in age, ethnicity, education, marital status, income, likelihood of smoking, mean alcohol use scores, illicit drug use in the past year, or marijuana use in the past year. Both groups demonstrated a significant increase in physical activity from baseline to year 1 follow-up.

\section{PTSD and Sleep Quality}

When sleep quality was regressed on group status in an unadjusted model, participants with current PTSD reported worse sleep quality (Table 2). When demographic variables (age, sex) were added to the model, PTSD status continued to be associated with worse sleep quality. When baseline physical activity, apnea risk, depression status, and BMI were added to the model, PTSD remained independently associated with worse sleep quality. Baseline physical activity and depression status were also associated with sleep quality 


\begin{tabular}{|c|c|c|c|}
\hline Variable & PTSD (n = 258) & No PTSD $(n=478)$ & $\mathrm{p}$ \\
\hline Age, years (SD) & $58.0(10.0)$ & $58.8(12.0)$ & 0.36 \\
\hline Male, $\mathrm{n}(\%)$ & $230(89 \%)$ & $464(97 \%)$ & $<0.001$ \\
\hline White, n (\%) & $152(59 \%)$ & $275(58 \%)$ & 0.72 \\
\hline High school education, $\mathrm{n}(\%)$ & $245(95 \%)$ & $462(97 \%)$ & 0.30 \\
\hline Married/partnered, n (\%) & $102(40 \%)$ & $162(34 \%)$ & 0.11 \\
\hline Annual household income $<\$ 20,000, n(\%)$ & $73(29 \%)$ & $159(33 \%)$ & 0.17 \\
\hline Depression, n (\%) & $152(60 \%)$ & $70(15 \%)$ & $<0.001$ \\
\hline Smoking status (yes), n (\%) & $64(24 \%)$ & $116(25 \%)$ & 0.78 \\
\hline Apnea probability (SD) & $0.54(0.23)$ & $0.48(0.21)$ & 0.001 \\
\hline Body mass index & $30.1(6.0)$ & $28.5(5.3)$ & $<0.001$ \\
\hline Alcohol use score (AUDIT-C) & $3.0(3.2)$ & $3.4(3.0)$ & 0.10 \\
\hline Illicit drug use (excluding marijuana) in past year, $n(\%)$ & $32(13 \%)$ & $48(10 \%)$ & 0.29 \\
\hline Marijuana use in past year, $\mathrm{n}(\%)$ & $71(28 \%)$ & $111(23 \%)$ & 0.16 \\
\hline Sleep quality, 0-4 (SD) & $1.4(0.9)$ & $2.2(1.0)$ & $<0.001$ \\
\hline Baseline physical activity (SD) & $2.2(1.7)$ & $2.8(1.5)$ & $<0.001$ \\
\hline Year 1 physical activity (SD) & $3.0(1.8)$ & $3.4(1.6)$ & 0.003 \\
\hline
\end{tabular}

Table 2-Sleep quality predicted by PTSD status

\begin{tabular}{|c|c|c|c|c|c|c|c|c|c|}
\hline & \multicolumn{3}{|c|}{ Model 1} & \multicolumn{3}{|c|}{ Model 2} & \multicolumn{3}{|c|}{ Model 3} \\
\hline & B & $\beta$ & SE & B & $\beta$ & SE & B & $\beta$ & SE \\
\hline PTSD status & $-0.805^{* * *}$ & -0.366 & 0.085 & $-0.831^{* * *}$ & -0.378 & 0.085 & $-0.514^{* *}$ & -0.234 & 0.093 \\
\hline Age at baseline & & & & $0.009 *$ & 0.101 & 0.003 & $0.009 *$ & 0.101 & 0.004 \\
\hline Sex & & & & $0.467^{*}$ & 0.100 & 0.183 & 0.323 & 0.069 & 0.195 \\
\hline Baseline physical activity & & & & & & & $0.084^{* * *}$ & 0.128 & 0.025 \\
\hline Apnea probability & & & & & & & -0.427 & -0.090 & 0.324 \\
\hline Depression status & & & & & & & $-0.571^{* * *}$ & -0.249 & 0.098 \\
\hline Body mass index & & & & & & & 0.010 & 0.047 & 0.012 \\
\hline $\mathrm{R}^{2}$ & & 0.134 & & & 0.151 & & & 0.228 & \\
\hline$\Delta \mathrm{R} 2$ (Step) & & 0.134 & & & 0.017 & & & 0.078 & \\
\hline
\end{tabular}

${ }^{*} p<0.05,{ }^{* *} p<0.01,{ }^{* * *} p<0.001$. Baseline physical activity was assessed using a 0-5 rating with higher scores indicative of more physical activity. Apnea probability was computed using a validated multivariate apnea risk index, which included self-report ratings of breathing-related behaviors, age, gender, and BMI. Depression status was evaluated using the Patient Health Questionnaire (PHQ-9).

\section{Predictors of Physical Activity One Year Later}

In unadjusted models, participants with current PTSD at baseline had lower physical activity one year later compared to those without PTSD one year later (Table 3). When demographic variables (age, sex) were added to the model, PTSD status continued to be associated with lower physical activity. When sleep quality was added to the model, PTSD was no longer independently associated with lower physical activity one year later. However, lower sleep quality predicted lower physical activity one year later. Hence, sleep was more strongly associated with physical activity one year later than was PTSD status. Finally, baseline physical activity, apnea probability, depression status, and BMI were added to the model. In this final model, sleep quality continued to be positively associated with physical activity one year later.

\section{Mediation Effects}

PTSD diagnosis was entered into the mediation model as a predictor, with baseline physical activity as a covariate.
Physical activity one year later was entered as the dependent variable, with baseline sleep quality entered as a possible mediator. There were significant direct effects whereby PTSD status was associated with worse sleep quality and baseline sleep quality predicted physical activity one year later (Figure 1). There was a significant indirect effect from PTSD status to physical activity one year later through baseline sleep quality. The direct effect from baseline PTSD status to physical activity one year later was not significant, indicating that sleep quality completely mediated the relationship between baseline PTSD status and physical activity one year later in this model (Figure 1).

While it is not possible to infer causality from the aforementioned mediation analysis given the concurrent measurement of sleep and PTSD, we ran the model in the reverse direction to substantiate our conclusions from the mediation analysis. In this analysis, baseline sleep quality was entered as a predictor. Physical activity one year later was entered as the dependent variable, with PTSD diagnosis entered as a possible mediator. 
Table 3-Predictors of year 1 physical activity

\begin{tabular}{|c|c|c|c|c|c|c|c|c|c|c|c|c|}
\hline & \multicolumn{3}{|c|}{ Model 1} & \multicolumn{3}{|c|}{ Model 2} & \multicolumn{3}{|c|}{ Model 3} & \multicolumn{3}{|c|}{ Model 4} \\
\hline & B & $\beta$ & SE & B & $\beta$ & SE & B & $\beta$ & SE & B & $\beta$ & SE \\
\hline PTSD status & $-0.371^{*}$ & -0.108 & 0.148 & $-0.387^{*}$ & -0.113 & 0.150 & -0.113 & -0.033 & 0.158 & 0.036 & 0.011 & 0.160 \\
\hline Age at baseline & & & & 0.008 & 0.056 & 0.006 & 0.004 & 0.032 & 0.006 & 0.004 & 0.031 & 0.007 \\
\hline Sex & & & & 0.386 & 0.053 & 0.324 & 0.203 & 0.028 & 0.321 & 0.263 & 0.036 & 0.330 \\
\hline Baseline sleep quality & & & & & & & $0.335^{\text {*** }}$ & 0.212 & 0.073 & $0.204^{* *}$ & 0.129 & 0.072 \\
\hline Baseline physical activity & & & & & & & & & & $0.353^{* * *}$ & 0.352 & 0.042 \\
\hline Apnea probability & & & & & & & & & & 0.158 & 0.021 & 0.539 \\
\hline Depression status & & & & & & & & & & -0.030 & -0.008 & 0.170 \\
\hline Body mass index & & & & & & & & & & -0.024 & -0.077 & 0.020 \\
\hline $\mathrm{R}^{2}$ & & 0.012 & & & 0.016 & & & 0.055 & & & 0.179 & \\
\hline$\Delta \mathrm{R} 2$ (Step) & & 0.012 & & & 0.005 & & & 0.038 & & & 0.125 & \\
\hline
\end{tabular}

${ }^{*} p<0.05,{ }^{* *} p<0.01,{ }^{* * *} p<0.001$. Sleep quality was assessed using a $0-4$ self-report rating and was coded such that higher scores indicated better sleep quality. Baseline physical activity was assessed using a 0-5 rating with higher scores indicative of more physical activity. Apnea probability was computed using a validated multivariate apnea risk index, which included self-report ratings of breathing-related behaviors, age, gender, and BMI. Depression status was evaluated using the Patient Health Questionnaire (PHQ-9).

This mediation model did not fit. There was a significant direct effect whereby sleep quality was associated with PTSD status. However, there was no significant direct effect of PTSD status to physical activity one year later, nor was there a significant indirect effect from sleep quality to physical activity one year later through PTSD status. The direct effect from sleep quality to physical activity one year later also was not significant.

\section{DISCUSSION}

In this cohort of 736 VA patients, sleep quality statistically mediated the association between PTSD and physical activity one year later. In addition, consistent with a large body of previous research, PTSD was associated with lower sleep quality after adjusting for potential confounders. ${ }^{23,45,46} \mathrm{We}$ also found that, unlike sleep quality, PTSD and depression did not independently predict physical activity in adjusted models.

Our finding that future physical activity was predicted by sleep quality at baseline provides some insight into the relationship between sleep and later physical activity. Previous research assessing the question of the relationship between sleep and physical activity has yielded mixed findings in healthy individuals, in which reduced sleep has been associated in various studies with less, more, or no change in physical activity. Several possibilities may explain the previous mixed findings on sleep and physical activity. For example, the mostly cross-sectional studies on the topic leave open the question of directionality between sleep and physical activity. That is, substantial evidence suggests that physical activity improves sleep ${ }^{47}$ but there has been a dearth of research on the other direction. The longitudinal association in the present study between sleep at baseline and physical activity one year later provides initial evidence that sleep may influence physical activity, consistent with other recent studies on this topic. ${ }^{8,10}$ The previous sleep-physical activity mixed findings may also relate to the short-term nature of experimental studies (i.e., acute sleep deprivation, next-day measurement of physical activity). The present study assessed self-reported
Figure 1-Sleep quality mediates the relationship between PTSD and physical activity at year 1 .

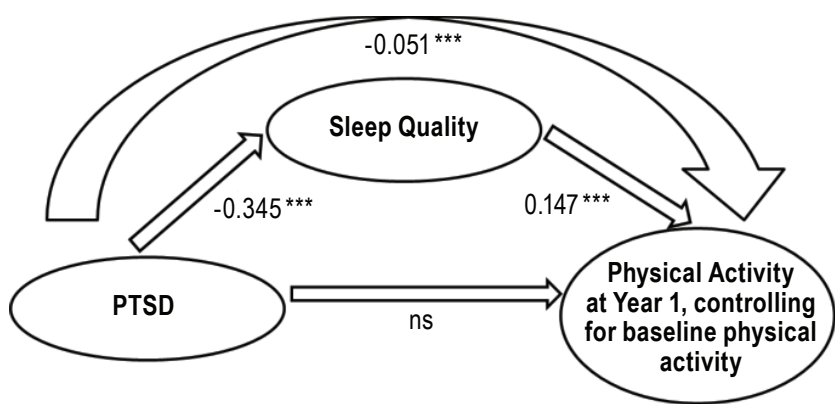

${ }^{* * *} p<0.001$. Baseline physical activity was included as a covariate.

sleep over the past month, and had a full year follow-up period for assessing habitual physical activity. Different samples may have also contributed to varying sleep-physical activity associations. For example, one study that found decreased sleep duration was associated with higher activity levels consisted of healthy adults in the Old Order Amish. The authors noted that this community was very physically active and such strenuous activity may be associated with increased wakefulness. ${ }^{48}$ Finally, few studies have examined sleep quality rather than duration. Our data regarding sleep quality and physical activity align with the one previous study on sleep quality, in which in a study of adolescents' sleep quality rather than sleep duration was associated with decreased physical activity measured using accelerometry. ${ }^{9}$

The finding that sleep quality predicts physical activity in patients with PTSD raises the possibility that behavioral factors could contribute to some of the poor physical health outcomes in PTSD such as obesity, metabolic syndrome, and coronary heart disease. . $6,17,19$ To date, most studies have investigated biological mechanisms through which sleep disturbance may cause negative health outcomes. ${ }^{20}$ However, initial research suggests that behaviors such as disinhibited eating in the context of poor sleep ${ }^{49}$ also appear to contribute to such health 
outcomes. The present study provides preliminary evidence that the association of reduced sleep quality with reduced physical activity could comprise another behavioral link to such health outcomes.

Clinically, the findings from the present study suggest that both low sleep quality and low physical activity are behavioral factors that should be assessed and potentially targeted in patients with PTSD, as we observed that individuals with PTSD reported worse sleep and lower physical activity at baseline compared to those without PTSD, consistent with previous research. ${ }^{23,25}$ Interestingly, physical activity for both the individuals with and without PTSD in this study increased from baseline to year one. The study did not include an intervention, but it is possible that participating in a study which focused on such topics may have influenced individuals' awareness of and engagement in health behaviors such as exercise. With regard to clinical interventions, the finding in the present study that sleep quality predicts later physical activity raises the possibility that sleep disturbance may need to be considered in interventions to increase physical activity. For example, sleep problems could affect individuals' willingness or ability to implement physical activity behavioral interventions, while sleep improvements might encourage exercise participation. ${ }^{8}$ Additionally, sleep quality might influence physical activity quality, such as effort expended during exercise or duration of exercise. Further research is needed to assess possible poor sleep-physical inactivity behavioral linkages.

Strengths of this study include its large sample size and longitudinal design. However, several limitations are important to consider. First, the assessments of sleep and physical activity were based on self-reported single-item measures. As such, participants may have misestimated their sleep quality or physical activity levels. However, we note that insomnia is currently defined based on self-report, ${ }^{50}$ and that single-item measures of sleep and physical activity have previously been associated with important psychological and medical outcomes. ${ }^{4,39}$ In particular, self-reported measures of sleep and physical activity have been predictors of metabolic syndrome $\mathrm{e}^{15}$ and cardiovascular events ${ }^{4}$ in previous studies. Nonetheless, we recommend that future research include longer and/or more frequent subjective assessments as well as objective assessments to examine physiological correlates of sleep quality and physical activity (i.e., objective measurement of fitness). Second, PTSD and sleep quality were measured concurrently; hence, we cannot assume that the pathway is from PTSD to reduced sleep quality rather than the opposite direction. However, we note that (a) there is substantial evidence for sleep disturbance occurring after the onset of trauma (although there is also some initial evidence suggesting that sleep disturbance may be a risk factor for PTSD), and (b) an exploratory analysis indicated that the mediation model in the opposite direction (i.e., PTSD mediating the effect between sleep quality and physical activity one year later) did not fit. Finally, the sample consisted primarily of male veterans, and as such the findings may not generalize to other groups.

In summary, our results demonstrate that PTSD is associated with lower sleep quality and that lower sleep quality is prospectively associated with lower physical activity. These results raise the possibility that improving sleep quality may be one component of increasing physical activity in PTSD.

\section{ABBREVIATIONS}

AUDIT-C, Alcohol Use Disorders Identification TestConsumption Questions

BMI, body mass index

CAPS, Clinician-Administered PTSD Scale

DSM-IV, Diagnostic and Statistical Manual of Mental

Disorders IV

PHQ-9, Patient Health Questionnaire

PSQI, Pittsburgh Sleep Quality Index

PTSD, posttraumatic stress disorder

VA, Department of Veterans Affairs

\section{REFERENCES}

1. Zschucke E, Gaudlitz K, Strohle A. Exercise and physical activity in mental disorders: Clinical and experimental evidence. J Prev Med Public Health 2013:46:S12-S21.

2. Oeland AM, Laessoe U, Olesen AV, Munk-Jorgensen P. Impact of exercise on patients with depression and anxiety. Nordic J Psychiat 2010;64:210-7.

3. Erickson KI, Voss MW, Prakash RS, et al. Exercise training increases size of hippocampus and improves memory. Proc Natl Acad Sci 2011;108:3017-22.

4. Whooley MA, de Jonge P, Vittinghoff E, et al. Depressive symptoms, health behaviors, and risk of cardiovascular events in patients with coronary heart disease. JAMA 2008:300:2379-88.

5. Ohida T, Kamal AM, Uchiyama M, et al. The influence of lifestyle and health status factors on sleep loss among the Japanese general population. Sleep 2001;24:333-38.

6. Driver HS, Taylor SR. Exercise and sleep. Sleep Med Rev 2000;4:387-402.

7. Imaki M, Hatanaka $Y$, Ogawa $Y$, Tanada S. An epidemiological study on relationship betwen the hours of sleep and life style factors in Japanese factory workers. J Physiol Anthropol Appl Human Sci 2002;21:115-20.

8. Baron KG, Reid KJ, Zee PC. Exercise to improve sleep in insomnia: exploration of the bidirectional effects. J Clin Sleep Med 2013;9:819-24.

9. Gupta NK, Mueller WH, Chan W, Meininger JC. Is obesity associated with poor sleep quality in adolescents? Am J Hum Biol 2002;14:762-68.

10. Penev PD. Update on energy homeostasis and insufficient sleep. $J$ Clin Endocrinol Metab 2012;97:1792-801.

11. Bromley LE, Booth JNI, Kilkus JM, Imperial JG, Penev PD. Sleep restriction decreases the physical activity of adults at risk for type 2 diabetes. Sleep 2012;35:977-84.

12. Buysse DJ. Sleep health: can we define it? Does it matter? Sleep 2014;37:9-17.

13. Krystal AD, Edinger JD. Measuring sleep quality. Sleep Med 2008;9 Suppl 1:S10-S17.

14. Hoevenaar-Blum MP, Spijkerman AM, Kromhout D, van der Berg JF, Verschuren WM. Sleep duration and sleep quality in relation to 12-year cardiovascular disease incidence: the MORGEN study. Sleep 2011;34:1487-92.

15. Jennings JR, Muldoon MF, Hall M, Buysse DJ, Manuck SB. Self-reported sleep quality is associated with the metabolic syndrome. Sleep 2007;30:219-23.

16. Patel SR, Hu FB. Short sleep duration and weight gain: A systematic review. Obesity 2012;16:643-53.

17. Troxel WM, Buysse DJ, Matthews KA, et al. Sleep symptoms predict the development of the metabolic syndrome. Sleep 2010;33:1633-40.

18. Vgontzas AN, Liao D, Bixler EO, Chrousos GP, Vela-Bueno A. Insomnia with objective short sleep duration is associated with a high risk for hypertension. Sleep 2009;32:491-7.

19. Chandola T, Ferrie JE, Perski A, Akbaraly T, Marmot MG. The effect of short sleep duration on coronary heart disease risk is greatest among those with sleep disturbance: a prospective study from the Whitehall II Cohort. Sleep 2010;33:739-44.

20. Spiegel K, Knutson K, Leproult R, Tasali E, Van Cauter E. Sleep loss: a novel risk factor for insulin resistance and Type 2 diabetes. J Appl Physiol 2005;99:2008-19.

21. Babson KA, Blonigen DM, Boden MT, Drescher KD, Boon-Miller MO. Sleep quality among U.S. Military Veterans with PTSD: A factor analysis and structural model of symptoms. J Trauma Stress 2012;25:665-74.

22. Krakow $B$, Hollifield $M$, Johnston $L$ et al. Imagery rehearsal therapy for chronic nightmares in sexual assault survivors with posttraumatic stress disorder: a randomized controlled trial. JAMA 2001;286:537-45. 
23. Roszell DK, McFall ME, Malas KL. Frequency of symptoms and concurrent psychiatric disorder in Vietnam veterans with chronic PTSD. Hosp Community Psychiatr 1991;42:293-96.

24. Germain A, Buysse DJ, Shear MK, Fayyad R, Austin C. Clinical correlates of poor sleep quality in posttraumatic stress disorder. J Trauma Stress 2004;17:474-84.

25. Chwastiak LA, Rosenheck RA, Kazis LE. Association of psychiatric illness and obesity, physical inactivity, and smoking among a national sample of veterans. Psychosomatics 2011;52:230-36.

26. Zen AL, Whooley MA, Zhao S, Cohen BE. Post-traumatic stress disorder is associated with poor health behaviors: findings from the Heart and Soul Study. Health Psychol 2012;31:194-201.

27. Buckley TC, Mozley SL, Bedard MA, Dewulf AC, Greif J. Preventive health behaviors, health-risk behaviors, physical morbidity, and health-related role functioning impairment in veterans iwth post-traumatic stress disorder. Mil Med 2004; 169:536-40.

28. de Assis MA, de Mello MF, Scorza FA, et al. Evaluation of physical activity habits in patients with posttraumatic stress disorder. Clinics (San Paulo) 2008;63:473-78.

29. Pagoto SL, Schneider KL, Bodenlos JS, et al. Association of post-traumatic stress disorder and obesity in a nationally representative sample. Obesity 2012;20:200-05.

30. Jin H, Lanouette NM, Mudaliar S, et al. Association of posttraumatic stress disorder with increased prevalence of metabolic syndrome. J Clin Psychopharm 2009:29:210-15.

31. Kubzansky LD, Koenen KC, Spiro Ar, Vokonas PS, Sparrow D. Prospective study of posttraumatic stress disorder symptoms and coronary heart disease in the Normative Aging Study. Arch Gen Psychiatry 2007;64:109-16.

32. Blake DD, Weathers FW, Nagy LM, et al. The development of a ClinicianAdministered PTSD Scale. J Trauma Stress 1995;8:75-90.

33. Weathers FW, Keane TM, Davidson JR. Clinician-administered PTSD Scale: a review of the first ten years of research. Depress Anxiety 2001;13:132-56.

34. Marshall RD, Olfson M, Hellman F, Blanco C, Guardino M, Struening EL. Comorbidity, impairment, and suicidality in subthreshold PTSD. Am J Psychiatry 2001;158:1467-73.

35. Blanchard EB, Hickling EJ, Taylor AE, Loos WR, Gerardi RJ. Psychological morbidity associated with motor vehicle accidents. Behav Res Ther 1994;32:283-90.

36. Buysse DJ, Reynolds CFd, Monk TH, Berman SR, Kupfer DJ. The Pittsburgh Sleep Quality Index: A new instrument for psychiatric practice and research. Psychiatr Res 1989;28:193-213.

37. Harvey AG, Stinson K, Whitaker KL, Moskovitz D, Virk H. The subjective meaning of sleep quality: a comparison of individuals with and without insomnia. Sleep 2008;31:383-93.

38. Buysse DJ, Ancoli-Israel S, Edinger JD, Lichstein KL, Morin CM. Recommendations for a standard research assessment of insomnia. Sleep 2006;29:1155-73.

39. Caska CM, Hendrickson BE, Wong MH, Ali S, Neylan T, Whooley MA. Anger expression and sleep quality in patients with coronary heart disease: Findings from the Heart and Soul study. Psychosom Med 2009;71:280-85.
40. Aadahl M, Kjaer M, Kristensen JH, Mollerup B, Jorgensen T. Self-reported physical activity compared iwth maximal oxygen update in adults. Eur J Cardiov Prev $R$ 2007; 14:422-28

41. Ainsworth BE, Jacobs DRJ, Leon AS. Validity and reliabilit of self-reported physical activity status: the Lipid Research Clinics questionnaire. Med Sci Sports Exerc 1993;25:92-98.

42. Maislin G, Pack Al, Kribbs NB, et al. A survey screen for prediction of apnea. Sleep 1994;18:158-66.

43. Bush K, Kivlahan DR, McDonell MB, Fihn SD, Bradley KA. The AUDIT Alcohol Consumption Questions (AUDIT-C): an effective brief screening test for problem drinking. Ambulatory Care Quality Improvement Project (ACQUIP). Alcohol Use Disorders Indentification Test. Arch Intern Med 1998;158:1789-95.

44. Kroenke K, Spitzer RL, Williams JBW. The PHQ-9: Validity of a brief depression severity measure. J Gen Intern Med 2001;16:606-13.

45. Ohayon MM, Shapiro CM. Sleep disturbances and psychiatric disorders associated with posttraumatic stress disorder in the general population. Compr Psychiatry 2000;41:469-78.

46. Neylan TC, Marmar CR, Metzler TJ, et al. Sleep disturbances in the Vietnam generation: findings from a nationally representative sample of male Vietnam veterans. Am J Psychiatry 1998;155:929-33.

47. Youngstedt SD, O'Connor PJ, Dishman RK. The effects of acute exercise on sleep: a quantitative synthesis. Sleep 1997;20:203-14.

48. Evans DS, Snitker S, Wu SH, et al. Habitual sleep/wake patterns in the Old Order Amish: heritability and association with non-genetic factors. Sleep 2011;34:661-69.

49. Chaput JP, Despres JP, Bouchard C, Tremblay A. The association between short sleep duration and weight gain is dependent on disinhibited eating behavior in adults. Sleep 2011;34:1291-97.

50. Edinger JD, Bonnet MH, Bootzin RR, et al. Derivation of research diagnostic criteria for insomnia: report of an American Academy of Sleep Medicine Work Group. Sleep 2004;27:1567-96.

\section{SUBMISSION \& CORRESPONDENCE INFORMATION}

Submitted for publication May, 2013

Submitted in final revised form February, 2014

Accepted for publication March, 2014

Address correspondence to: Lisa S. Talbot, San Francisco VA Medical Center (116-H), 4150 Clement St., San Francisco, CA 94121; Tel: (650) 799-0810; Email: lisa.talbot@ gmail.com

\section{DISCLOSURE STATEMENT}

This was not an industry supported study. This research was supported by grants from the National Heart, Lung, and Blood Institute (BEC: K23 HL 094765), the Irene Perstein Foundation, and the Mental IIIness Research and Education Clinical Center of the US Veterans Health Administration. The authors have indicated no financial conflicts of interest. 